\title{
Diatomáceas (Bacillariophyceae) em marismas no sul do Brasil
}

\author{
Juliana Gonçalves da Silva ${ }^{1,3}$, Lezilda Carvalho Torgan ${ }^{1}$ e Luciana de Souza Cardoso ${ }^{2}$
}

Recebido em 12/11/2009. Aceito em 19/08/2010

RESUMO - (Diatomáceas (Bacillariophyceae) em marismas no sul do Brasil). Nas marismas do sul do Brasil a comunidade de diatomáceas é desconhecida. Este estudo visa avaliar a composição, a riqueza e a $\beta$-diversidade desta comunidade com base em amostras coletadas em cinco pontos georeferenciados, entre as coordenadas $31^{\circ} 59^{\prime} 18.60^{\prime \prime} \mathrm{S}-52^{\circ} 14^{\prime} 58.50^{\prime \prime} \mathrm{W}$ e $32^{\circ} 06^{\prime} 49.74^{\prime \prime} \mathrm{S}-52^{\circ} 09^{\prime} 22.57^{\prime \prime} \mathrm{W}$, em janeiro de 2002 e março de 2008, no estuário da laguna dos Patos. Esta comunidade esteve composta por $64 \%$ de diatomáceas salobras, $24 \%$ de marinhas e $16 \%$ de dulcícolas e esteve representada por 24 famílias, 36 gêneros e 50 táxons específicos e intra-específicos. Não houve variação na composição das espécies indicada pela diversidade- $\beta(1,05)$. Com base na estimativa de riqueza Chao2, a comunidade de diatomáceas se mostrou representativa das marismas do estuário da laguna dos Patos. As espécies identificadas são apresentadas com suas medidas e ilustrações.

Palavras-chave: composição florística, riqueza, subtropical, $\beta$-diversidade

ABSTRACT - (Salt Marsh Diatoms (Bacillariophyceae) in South Brazil). Salt marshes in South Brazil hold an unknown diatom community. This study aims to evaluate the composition, richness and $\beta$-diversity of the diatom community of salt marsh sediments in the Patos Lagoon Estuary. The samples were taken at five sites between $31^{\circ} 59^{\prime} 18.60^{\prime \prime} \mathrm{S}-52^{\circ} 14^{\prime} 58.50^{\prime \prime} \mathrm{W}$ and $32^{\circ} 06^{\prime} 49.74^{\prime \prime} \mathrm{S}-52^{\circ} 09^{\prime} 22.57^{\prime \prime} \mathrm{W}$, in January 2002 and March 2008 . The community was composed of $64 \%$ brackish, $24 \%$ marine and $16 \%$ freshwater diatoms and it was represented by 24 families, 36 genera and 50 specific and infra-specific taxa. There was no variation in the species composition indicated by $\beta$-diversity (1.05). Based on the Chao 2 richness estimate, the diatom community is representative of the salt marsh in the Patos Lagoon Estuary. Measurements and illustrations of the species are presented.

Key words: floristic composition, richness, subtropical, $\beta$-diversity

\section{Introdução}

No Brasil, as marismas dominam a paisagem de margens sedimentares em estuários, lagunas e baías dos estados de Santa Catarina e do Rio Grande do Sul. Cerca de $70 \mathrm{~km}^{2}$ das margens e ilhas do estuário da laguna dos Patos (RS) são recobertos por marismas.

As diatomáceas auxiliam na estabilização do sedimento das marismas, por outro lado, a variedade de habitats relacionada à heterogênea altura topográfica das marismas (planos de lama, planos vegetados, canais e poças-de-maré) incrementa a diversidade do grupo. Segundo Round et al. (1990), as diatomáceas presentes no sedimento representam bem a diversidade deste grupo no sistema, pois este meio integra espacialmente táxons provindos do perifíton, plâncton e bentos.

Nas águas do estuário da laguna dos Patos, observamse grandes variações de temperatura e salinidade, que são determinadas principalmente pela ação dos ventos, pela temperatura do ar e pelo regime de chuvas atuantes no período (Vilas Boas apud Seeliger \& Costa, 1998). Em função desta dinâmica, pode-se observar grande flutuação na composição química da água, que tende a variar em função da mudança de salinidade (Niencheski \& Windom, 1994).

A atual configuração geomorfológica do estuário da laguna dos Patos é conseqüente de processos erosivos e deposicionais resultantes da ação morfodinâmica Holocênica (Villwock et al., 1986). A análise diatomológica é amplamente utilizada na interface de ambientes dulcícolas e salinos para identificar lagos isolados do mar em áreas de soerguimento, indicando transgressão de águas marinha e salobra, e localizar a posição da linha de litoral no passado (Battarbee, 1986).
Além disso, a comunidade de diatomáceas, através de atributos como diversidade de espécies e abundância de indivíduos, é uma excelente ferramenta para a gestão de alterações ambientais sobre os ecossistemas decorrentes de ações antropogênicas, funcionando como indicadoras de qualidade ambiental.

Nos últimos 150 anos, as marismas do município de Rio Grande têm sido gradativamente destruídas, aterradas e transformadas em área urbana (Seeliger \& Costa, 1998), tornando urgente estudos básicos para conhecimento e conservação das comunidades que habitam este ambiente.

Nas marismas do sul do Brasil a comunidade diatomológica não é conhecida. Este trabalho pretende contribuir pioneiramente para o conhecimento de diatomáceas nos sedimentos de marisma tendo como objetivo avaliar a composição, estimar a riqueza e conhecer a sua distribuição.

\section{Material e métodos}

As amostragens foram efetuadas na margem leste do estuário da laguna dos Patos, nas proximidades do município de Rio Grande (Fig. 1), em cinco pontos georreferenciados. A área é caracterizada por faixas vegetadas, principalmente, por Spartina alterniflora Lois., Scirpus olneyi A. Gray, Scirpus maritimus L. e Spartina densiflora Brongn. (Cordazzo \& Seeliger, 1995).

Sedimentos superficiais foram coletados com espátula em profundidades de 1 a $3 \mathrm{~cm}$. Para tal, efetuaram-se duas expedições; na primeira, realizada em janeiro de 2002, coletou-se nos pontos 1 e 2; na segunda, realizada em de março de 2008, nos pontos 3, 4 e 5 (Tab. 1). Mensurações pontuais de temperatura, condutividade, salinidade e $\mathrm{pH}$ foram realizadas in situ, através de aparelhos HACH modelos 50150 e 50050.

As amostras foram oxidadas pelo método descrito por Van Der Werff (1955) e montadas em lâminas com a resina Naphrax como meio de inclusão. As diatomáceas foram observadas e fotografadas ao microscópio Zeiss Axioplan. Para a identificação das espécies e informações do habitat foram utilizadas as seguintes referências bibliográficas: Van Heurck (1896), Frenguelli (1929), Hustedt (1930), Frenguelli (1935), Hustedt (1937),

\footnotetext{
Fundação Zoobotânica do Rio Grande do Sul. Museu de Ciências Naturais, Porto Alegre, RS, Brasil

2 Universidade Federal do Rio Grande do Sul. Departamento de Botânica, Porto Alegre, RS, Brasil

3 Autor para correspondência: biologa.juliana@gmail.com
} 


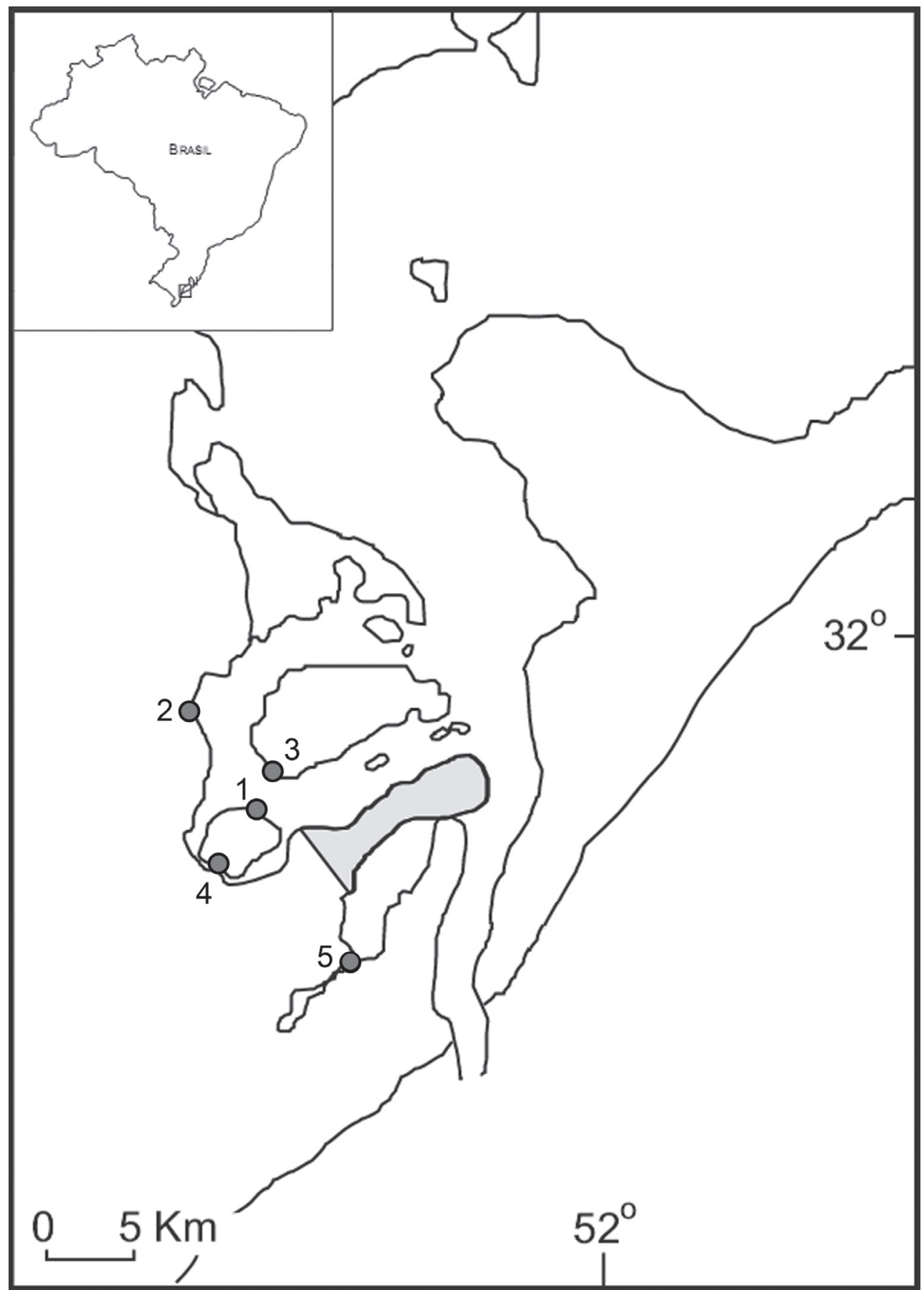

Figura 1. Localização dos pontos amostrados nas marismas do estuário da laguna dos Patos. (Rio Grande, RS, Brasil).

Tabela 1. Relação dos pontos com suas respectivas datas, coletores, coordenadas geográficas e registro no Herbário Prof. Dr. Alarich Schultz (HAS).

\begin{tabular}{|c|c|c|c|c|}
\hline Estações & Data & Coletor & Coordenadas Geográficas & Registro no HAS \\
\hline 1 & $27 / \mathrm{I} / 2002$ & Medeanic s.n. & $32^{\circ} 02^{\prime} 21.00^{\prime \prime} \mathrm{S} ; 52^{\circ} 12^{\prime} 42.00^{\prime \prime} \mathrm{O}$ & HAS 107873 \\
\hline 2 & $27 / \mathrm{I} / 2002$ & Medeanic s.n. & $31^{\circ} 59^{\prime} 18.60^{\prime \prime} \mathrm{S} ; 52^{\circ} 14^{\prime} 58.50^{\prime \prime} \mathrm{O}$ & HAS 107874 \\
\hline 3 & $27 / \mathrm{III} / 2008$ & Torgan s.n. & $32^{\circ} 01^{\prime} 37.15^{\prime \prime} \mathrm{S} ; 52^{\circ} 11^{\prime} 44.45^{\prime \prime} \mathrm{O}$ & HAS 107850 \\
\hline 4 & $27 / \mathrm{III} / 2008$ & Torgan s.n. & $32^{\circ} 03^{\prime} 17.67^{\prime \prime} \mathrm{S} ; 52^{\circ} 14^{\prime} 45.93^{\prime \prime} \mathrm{O}$ & HAS 107851 \\
\hline 5 & 27/III/2008 & Torgan s.n. & $32^{\circ} 06^{\prime} 49.74^{\prime \prime} \mathrm{S} ; 52^{\circ} 09^{\prime} 22.57^{\prime \prime} \mathrm{O}$ & HAS 107853 \\
\hline
\end{tabular}


Frenguelli (1938), Hendey (1964), Hustedt (1966), Patrick \& Reimer (1966), Rosa (1982), Krammer \& Lange-Bertalot (1985), Buselato-Toniolli (1986), Krammer \& Lange-Bertalot (1986), Sündback \& Medlin (1986), Krammer \& Lange-Bertalot (1988), Moreira-Filho (1990), Round et al. (1990), Snoeijs et al. (1991), Snoeijs (1992), Hartley (1996), Metzeltin \& Witkowski (1996), Lange-Bertalot (1998), Witkowski et al. (2000), Lange-Bertalot (2001), Danielidis \& Mann (2003), Metzeltin et al. (2005), Siver et al. (2005).

O material estudado encontra-se tombado no Herbário Prof. Dr. Alarich Schultz (HAS) no Museu de Ciências Naturais da Fundação Zoobotânica do Rio Grande do Sul (Tab. 1).

Para tornar os dados obtidos comparáveis a outros inventários e, também, avaliar o esforço amostral estimou-se a riqueza de espécies da comunidade amostrada pela segunda aproximação de Chao (Chao, 1987) através do software Estimates versão 8.0 (Colwell, 2006).

Medidas de heterogeneidade da comunidade de diatomáceas da marisma foram obtidas através do cálculo da diversidade- $\beta$ (Whittaker, 1972) que revela heterogeneidades no gradiente espacial estudado através das diferentes associações de espécies nos habitats, assumidos neste estudo como os diferentes pontos amostrados. Este índice mede como as comunidades se alteram ao longo de gradientes ambientais (Whittaker, 1972). O índice utilizado foi o $\beta \mathrm{w}$ (Whittaker, 1960) através da fórmula $\beta \mathrm{w}=(\mathrm{S} / \alpha$ médio $)-1$ na qual, "S" é número total de espécies dos pontos amostrados, " $\alpha$ médio" é número médio de espécies do grupo de pontos amostrais.

\section{Resultados e discussão}

No sedimento das marismas do estuário da laguna dos Patos, foram registrados 50 táxons específicos e infraespecíficos de diatomáceas, distribuídos em 24 famílias e 36 gêneros. Dentre estes 15 espécies são novos registros para o Estado. Os taxóns são apresentados a seguir.

Classe Coscinodiscophyceae

Ordem Thalassiosirales

Família Stephanodiscaceae

Cyclotella meneghiniana Kützing, 1844

Fig. 2A

Medidas: diâmetro 6-13 $\mu \mathrm{m}$. Habitat: marinho e dulcícola.

Material examinado: BRASIL. Rio Grande do Sul: Rio Grande, laguna dos Patos, (HAS 107873, lâm. 6045, 6048); (HAS 107851, lâm. 6067); (HAS 107853, lâm. 6070).

Cyclotella striata (Kützing) Grunow, 1880

Fig. 2B

Medidas: diâmetro 21-25 $\mu \mathrm{m}$. Habitat: dulcícola e salobro.

Material examinado: BRASIL. Rio Grande do Sul: Rio Grande, laguna dos Patos, (HAS 107851, lâm n. 6067); (HAS 107853, lâm n. 6070).

Ordem Paraliales

Família Paraliaceae

Paralia sulcata (Ehrenberg) Cleve, 1873

Fig. 2C

Medidas: diâmetro 18-31 $\mu \mathrm{m}$. Habitat: marinho.

Material examinado: BRASIL. Rio Grande do Sul: Rio Grande, laguna dos Patos, (HAS 107873, lâm n. 6045, 6049).
Ordem Coscinodiscales

Família Heliopeltaceae

Actinoptychus senarius (Ehrenberg) Ehrenberg, 1843

Fig. 2D

Medidas: diâmetro $19 \mu \mathrm{m}$. Habitat: marinho.

Material examinado: BRASIL. Rio Grande do Sul:

Rio Grande, laguna dos Patos, (HAS 107873, lâm n. 6048).

Ordem Triceratiales

Família Triceratiaceae

Pleurosira laevis (Ehrenberg) Compère, 1982

Fig. 2E

Medidas: comprimento 44-75 $\mu \mathrm{m}$. Habitat: salobro.

Material examinado: BRASIL. Rio Grande do Sul: Rio Grande, laguna dos Patos, (HAS 107873, lâm n. 6048); (HAS 107851, lâm n. 6066).

Família Plagiogrammaceae

Dimeregramma minor (Gregory) Ralfs, 1861

Fig. 2F

Medidas: comprimento $24 \mu \mathrm{m}$; largura $8 \mu \mathrm{m}$; 13-14 estrias em $10 \mu \mathrm{m}$. Habitat: marinho.

Material examinado: BRASIL. Rio Grande do Sul: Rio Grande, laguna dos Patos, (HAS 107874 , lâm n. 6056).

Ordem Biddulphiales

Família Biddulphiaceae

Terpsinoë americana (Bailey) Grunow, 1868

Fig. $2 \mathrm{G}$

Medidas: comprimento 38-45 $\mu \mathrm{m}$; largura 29-32 $\mu \mathrm{m}$; 3 costas em $10 \mu \mathrm{m}$. Habitat: salobro.

Material examinado: BRASIL. Rio Grande do Sul: Rio Grande, laguna dos Patos, (HAS 107873, lâm n. 6048); (HAS 107850, lâm n. 6061, 6062); (HAS 107853, lâm n. 6071).

Ordem Cymatosirales

Família Cymatosiraceae

Cymatosira belgica Grunow, 1881

Fig. $2 \mathrm{H}$

Medidas: comprimento $14 \mu \mathrm{m}$; largura $3 \mu \mathrm{m}$; 7 estrias em $10 \mu \mathrm{m}$. Habitat: marinho.

Material examinado: BRASIL. Rio Grande do Sul: Rio Grande, laguna dos Patos, (HAS 107874, lâm n. 6058).

Classe Fragillariophyceae

Ordem Fragillariales

Família Fragillariaceae

Martyana martyi (Héribaud) Round, 1990

Fig. 2I

Medidas: comprimento $10 \mu \mathrm{m}$; largura $4 \mu \mathrm{m}$ de largura. Habitat: dulcícola. 
Material examinado: BRASIL. Rio Grande do Sul: Rio Grande, laguna dos Patos, (HAS 107873, lâm n. 6048); (HAS 107874, lâm n. 6056); (HAS 107853, lâm n. 6070).

Tabularia fasciculata (Agardh) Snoeijs, 1986 Fig. 2J

Medidas: comprimento 55-70 $\mu \mathrm{m}$; largura $5 \mu \mathrm{m}$ de largura. Habitat: salobro.

Material examinado: BRASIL. Rio Grande do Sul: Rio Grande, laguna dos Patos, (HAS 107873, lâm n. 6048); (HAS 107851, lâm n. 6067).

Classe Bacillariophyceae

Ordem Mastogloiales

Família Mastogloiacae

Mastogloia pumila (Grunow) Cleve, 1895

Fig. 2K

Medidas: comprimento 27-35 $\mu \mathrm{m}$; largura 8,5-9 $\mu \mathrm{m} ; 27$ estrias em $10 \mu \mathrm{m}$. Habitat: salobro e marinho.

Material examinado: BRASIL. Rio Grande do Sul: Rio Grande, laguna dos Patos, (HAS 107850, lâm n. 6061); (HAS 107851, lâm n. 6067).

Ordem Cymbellales

Família Anomoeoneidaceae

Staurophora salina (Smith) Mereschkowsky, 1903

Fig. 2L

Medidas: comprimento 25-55 $\mu \mathrm{m}$; largura 11-12 $\mu \mathrm{m}$; 18 estrias em $10 \mu \mathrm{m}$. Habitat: marinho.

Material examinado: BRASIL. Rio Grande do Sul: Rio Grande, laguna dos Patos, (HAS 107850, lâm n. 6061); (HAS 107851, lâm n. 6067); (HAS 107853, lâm n. 6071).

Família Cymbellaceae

Cymbella grossestriata Müller, 1905

Fig. 2M

Medidas: comprimento 81-91 $\mu \mathrm{m}$; largura 14-15 $\mu \mathrm{m}$; 7 estrias em $10 \mu \mathrm{m}$. Habitat: salobro.

Primeira citação de ocorrência da espécie para o Estado.

Material examinado: BRASIL. Rio Grande do Sul: Rio Grande, laguna dos Patos, (HAS 107873, lâm n. 6045); (HAS 107850, lâm n. 6063); (HAS 107851, lâm n. 6066).

Placoneis abundans Metzeltin, Lange-Bertalot \& GarcíaRodriguez, 2005

Fig. 2N

Medidas: comprimento $28 \mu \mathrm{m}$; largura $11 \mu \mathrm{m}$; 12 estrias em $10 \mu \mathrm{m}$. Habitat: dulcícola.

Primeira citação de ocorrência da espécie para o Estado.

Material examinado: BRASIL. Rio Grande do Sul: Rio Grande, laguna dos Patos, (HAS 107873, lâm n. 6045).
Ordem Achnanthales

Família Achnanthaceae

Achnanthes curvirostrum Brun, 1895

Fig. $2 \mathrm{O}$

Medidas: comprimento 37-40 $\mu \mathrm{m}$; largura 14-15 $\mu \mathrm{m}$; 12-13 estrias em $10 \mu \mathrm{m}$. Habitat: salobro.

Material examinado: BRASIL. Rio Grande do Sul: Rio Grande, laguna dos Patos, (HAS 107850, lâm n. 6062); (HAS 107851, lâm n. 6067); (HAS 107853, lâm n. 6070).

Achnanthes parvula Kützing, 1844

Fig. 2P

Medidas: comprimento 30-47 $\mu \mathrm{m}$; largura 8-10 $\mu \mathrm{m}$; 9-10 estrias em $10 \mu \mathrm{m}$. Habitat: marinho.

Primeira citação de ocorrência da espécie para o Estado.

Material examinado: BRASIL. Rio Grande do Sul: Rio Grande, laguna dos Patos, (HAS 107873, lâm n. 6044, 6046, 6048, 6049); (HAS 107874, lâm n. 6056).

Família Cocconeidaceae

Cocconeis disculoides Hustedt, 1955

Fig. 3A

Medidas: comprimento 13-16 $\mu \mathrm{m}$; largura $8-9 \mu \mathrm{m}$; 8 linhas de estrias em $10 \mu \mathrm{m}$. Habitat: salobro.

Material examinado: BRASIL. Rio Grande do Sul: Rio Grande, laguna dos Patos, (HAS 107873, lâm n. 6046); (HAS 107850, lâm n. 6061, 6062); (HAS 107853, lâm n. 6070).

Cocconeis placentula var. lineata (Ehrenberg) Van Heurck, 1885

Fig. 3B

Medidas: comprimento 18-34 $\mu \mathrm{m}$; largura 9,5-21 $\mu \mathrm{m}$; 8 linhas de estrias em $10 \mu \mathrm{m}$. Habitat: salobro.

Material examinado: BRASIL. Rio Grande do Sul: Rio Grande, laguna dos Patos, (HAS 107850, lâm n. 6062); (HAS 107851, lâm n. 6066); (HAS 107853, lâm n. 6070).

Ordem Naviculales

Família Berkeleyaceae

Parlibellus crucicula (Smith) Witkowski, Lange-Bertalot \& Metzeltin, 2000

Fig. 3C

Medidas: comprimento 36-39 $\mu \mathrm{m}$; largura $11 \mu \mathrm{m}$; 19 estrias em $10 \mu \mathrm{m}$. Habitat: dulcícola e salobro.

Primeira citação de ocorrência da espécie para o Estado.

Material examinado: BRASIL. Rio Grande do Sul: Rio Grande, laguna dos Patos, (HAS 107873, lâm n. 6048).

Família Diasdesmidaceae

Luticola goeppertiana (Bleisch) Mann, 1990

Fig. 3D

Medidas: comprimento 15-20 $\mu \mathrm{m}$; largura 6-8 $\mu \mathrm{m} ; 15$ estrias em $10 \mu \mathrm{m}$. Habitat: salobro e dulcícola. 

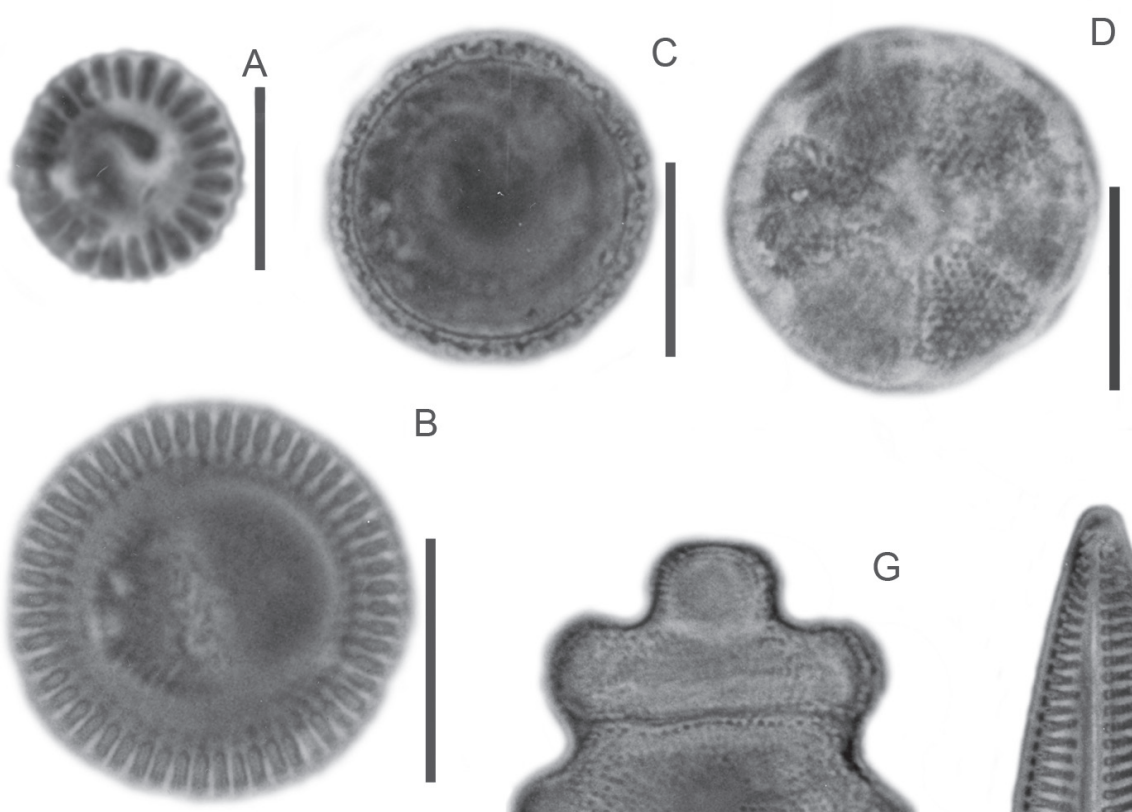

B
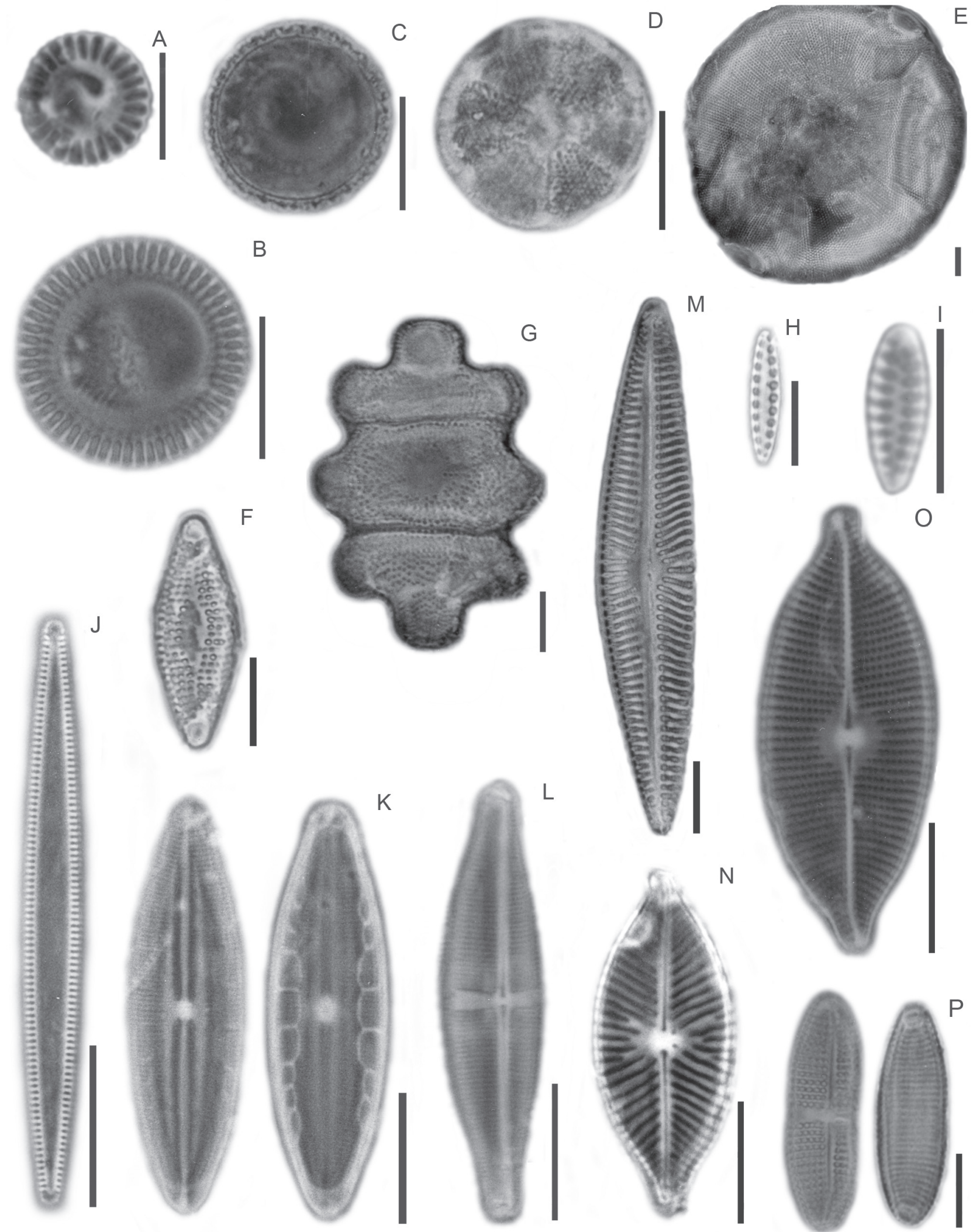

Figura 2. A. Cyclotella meneghiniana Kützing. B. Cyclotella striata Kützing (Grunow). C. Paralia sulcata (Ehrenberg) Cleve. D. Actinoptychus senarius (Ehrenberg) Ehrenberg. E. Pleurosira laevis (Ehrenberg) Compère. F. Dimeregramma minor (Gregory) Ralfs. G. Terpsinoë americana (Bailey) Grunow. H. Cymatosira belgica Grunow. I. Martyana martyi (Héribaud) Round. J. Tabularia fasciculata (Agardh) Snoeijs. K. Mastogloia pumila (Grunow) Cleve. L. Staurophora salina (Smith) Mereschkowsky. M. Cymbella grossestriata Müller. N. Placoneis abundans Metzeltin, Lange-Bertalot \& García-Rodriguez. O. Achnanthes curvirostrum Brun. P. Achnanthes parvula Kützing. Barra $=10 \mu \mathrm{m}$. 
Material examinado: BRASIL. Rio Grande do Sul: Rio Grande, laguna dos Patos, (HAS 107873, lâm n. 6044); (HAS 107874, lâm n. 6058); (HAS 107850, lâm n. 6061); (HAS 107851, lâm n. 6067) (HAS 107853, lâm n. 6070).

Família Amphipleuraceae

Frustulia creuzburgensis (Krasske) Hustedt, 1957

Fig. 3E

Medidas: comprimento $32 \mu \mathrm{m}$; largura $7 \mu \mathrm{m}$ de largura. Habitat: salobro e dulcícola.

Primeira citação de ocorrência da espécie para o Estado.

Material examinado: BRASIL. Rio Grande do Sul: Rio Grande, laguna dos Patos, (HAS 107850, lâm n. 6061).

Família Sellaphoraceae

Fallacia oculiformis (Hustedt) Mann, 1990

Fig. 3F

Medidas: comprimento 7-10 $\mu \mathrm{m}$; largura 4-6 $\mu \mathrm{m}$ de largura. Habitat: salobro e marinho.

Primeira citação de ocorrência da espécie para o Estado.

Material examinado: BRASIL. Rio Grande do Sul: Rio Grande, laguna dos Patos, (HAS 107850, lâm n. 6061, 6062).

Família Pinnulariaceae

Caloneis bivittata var. lata Heiden, 1906

Fig. $3 \mathrm{G}$

Medidas: comprimento $143 \mu \mathrm{m}$; largura $49 \mu \mathrm{m}$; 9 estrias em $10 \mu \mathrm{m}$. Habitat: salobro.

Material examinado: BRASIL. Rio Grande do Sul: Rio Grande, laguna dos Patos, (HAS 107873, lâm n. 6048).

Família Diploneidaceae

Diploneis didyma (Ehrenberg) Cleve, 1894

Fig. $3 \mathrm{H}$

Medidas: comprimento 39-61 $\mu \mathrm{m}$; largura 20-26 $\mu \mathrm{m}$; 8 estrias em $10 \mu \mathrm{m}$. Habitat: salobro.

Material examinado: BRASIL. Rio Grande do Sul: Rio Grande, laguna dos Patos, (HAS 107873, lâm n. 6046); (HAS 107851, lâm n. 6067); (HAS 107853, lâm n. 6070).

Diploneis smithii (Brébisson) Cleve, 1894

Fig. 3I

Medidas: comprimento 29-64 $\mu \mathrm{m}$; largura 26-40 $\mu \mathrm{m}$; 5-6 estrias em $10 \mu \mathrm{m}$. Habitat: salobro e marinho.

Material examinado: BRASIL. Rio Grande do Sul: Rio Grande, laguna dos Patos, (HAS 107873, lâm n. 6045, 6048); (HAS 107850, lâm n.6061); (HAS 107851, lâm n. 6067); (HAS 107853, lâm n. 6070).

Família Naviculaceae

Capartogramma crucicula (Grunow) Ross, 1963

Fig. 3J
Medidas: $34 \mu \mathrm{m}$; largura $12 \mu \mathrm{m}$; 25-26 estrias em 10 $\mu \mathrm{m}$. Habitat: salobro.

Material examinado: BRASIL. Rio Grande do Sul: Rio Grande, laguna dos Patos,

Navicula peregrina (Ehrenberg) Kützing, 1844

Fig. 3K

Medidas: comprimento $88 \mu \mathrm{m}$; largura $16 \mu \mathrm{m}$; 7 estrias em $10 \mu \mathrm{m}$. Habitat: salobro.

Material examinado: BRASIL. Rio Grande do Sul: Rio Grande, laguna dos Patos, (HAS 107853, lâm n. 6070).

Navicula recens (Lange-Bertalot) Lange-Bertalot, 1985

Fig. 3L

Medidas: comprimento 41-51 $\mu \mathrm{m}$; largura 7-10 $\mu \mathrm{m}$; 9-11 estrias em $10 \mu \mathrm{m}$. Habitat: salobro.

Material examinado: BRASIL. Rio Grande do Sul: Rio Grande, laguna dos Patos, (HAS 107873, lâm n. 6045); (HAS 107850, lâm n. 6061, 6062); (HAS 107851, lâm n. 6067); (HAS 107853, lâm n. 6071).

Seminavis strigosa (Hustedt) Danieldis \& EconomouAmilli, 2003

Fig. 3M

Medidas: comprimento 25-30 $\mu \mathrm{m}$; largura 4-5 $\mu \mathrm{m}$; 16 estrias em $10 \mu \mathrm{m}$. Habitat: salobro.

Primeira citação de ocorrência da espécie para o Estado.

Material examinado: BRASIL. Rio Grande do Sul: Rio Grande, laguna dos Patos, (HAS 107850, lâm n. 6062).

Chamaepinnularia truncata (Dieter König) Witkowski, Lange-Bertalot \& Metzeltin, 2000

Fig. $3 \mathrm{~N}$

Medidas: comprimento 15-20 $\mu \mathrm{m}$; largura 6,5-7 $\mu \mathrm{m}$; 18-19 estrias. Habitat: salobro.

Primeira citação de ocorrência da espécie para o Estado.

Material examinado: BRASIL. Rio Grande do Sul: Rio Grande, laguna dos Patos, (HAS 107850, lâm n. 6061, 6062).

Hippodonta hungarica (Grunow) Lange-Bertalot, Metzeltin \& Witkowski, 1996

Fig. 30

Medidas: comprimento 15-21 $\mu \mathrm{m}$; largura $6 \mu \mathrm{m}$ de largura. Habitat: dulcícola.

Material examinado: BRASIL. Rio Grande do Sul: Rio Grande, laguna dos Patos, (HAS 107851, lâm n. 6067); (HAS 107853, lâm n. 6070).

Ordem Thalassiophysales

Família Catenulaceae

Catenula adhaerens (Mereschkowsky) Mereschkowsky, 1902 Fig. 4A 


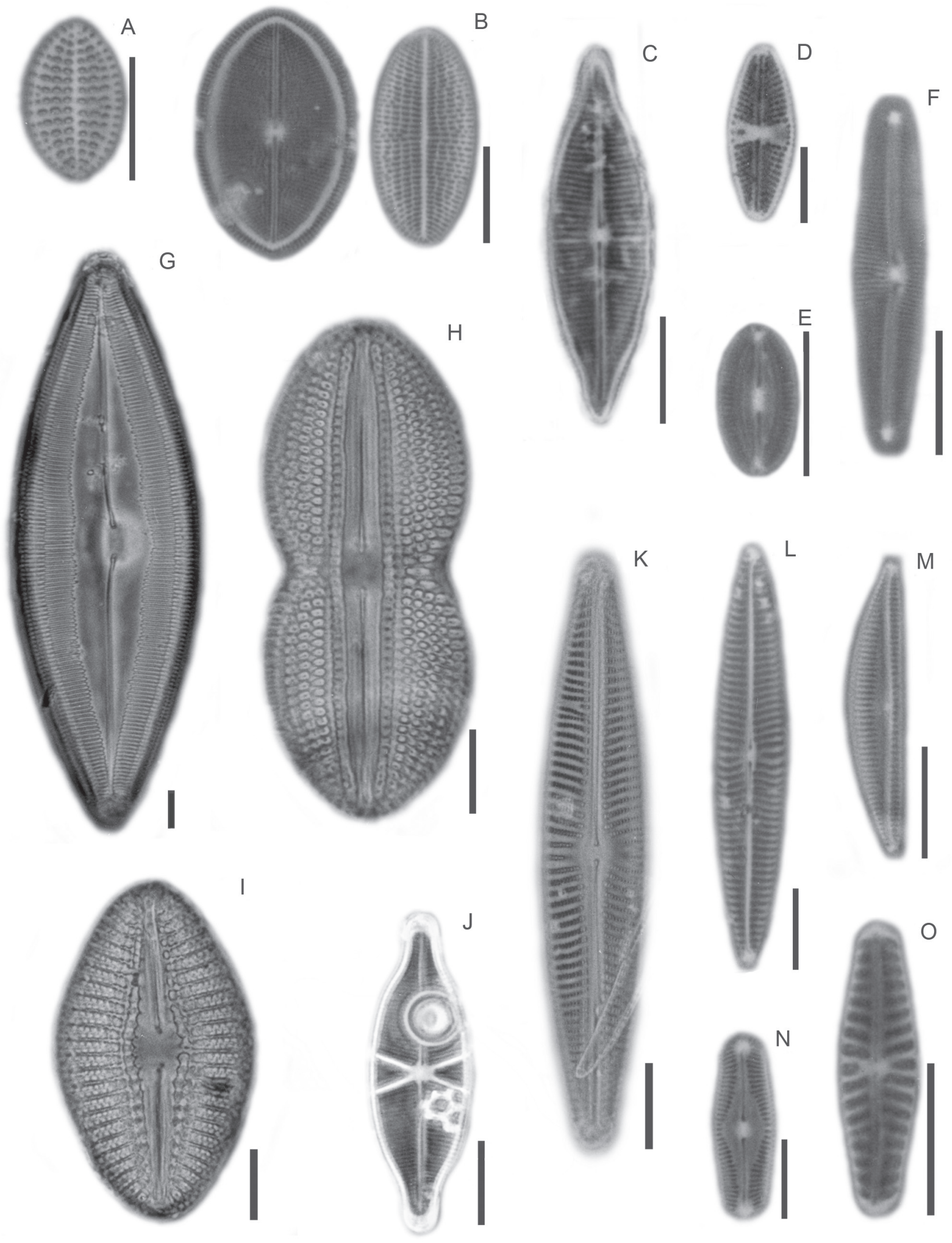

Figura 3. A. Cocconeis disculoides Hustedt. B. Cocconeis placentula var. lineata (Ehrenberg) Van Heurck. C. Parlibellus crucicula (Smith) Witkowski, Lange-Bertalot \& Metzeltin. D. Luticola goeppertiana (Bleisch) Mann. E. Frustulia creuzburgensis (Krasske) Hustedt. F. Fallacia oculiformis (Hustedt) Mann. G. Caloneis bivittata var. lata Heiden. H. Diploneis didyma (Ehrenberg) Cleve. I. Diploneis smithii (Brébisson) Cleve. J. Capartogramma crucicula (Grunow) Ross. K. Navicula peregrina (Ehrenberg) Kützing. L. Navicula recens (Lange-Bertalot) Lange-Bertalot. M. Seminavis strigosa (Hustedt) Danieldis \& Economou-Amilli. N. Chamaepinnularia truncata (Dieter König) Witkowski, Lange-Bertalot \& Metzeltin. O. Hippodonta hungarica (Grunow) Lange-Bertalot, Metzeltin \& Witkowski. Barra $=10 \mu \mathrm{m}$. 
Medidas: comprimento 11-15 $\mu \mathrm{m}$; largura 2-5 $\mu \mathrm{m}$ de largura. Habitat: salobro e marinho.

Primeira citação de ocorrência da espécie para o Estado.

Material examinado: BRASIL. Rio Grande do Sul: Rio Grande, laguna dos Patos, (HAS 107873, lâm n. 6046); (HAS 107874, lâm n. 6056, 6058); (HAS 107850, lâm n. 6062); (HAS 107853, lâm n. 6070).

\section{Amphora crucifera Cleve, 1922}

Fig. 4B

Medidas: comprimento 44-55 $\mu \mathrm{m}$; largura 9-10 $\mu \mathrm{m} ; 13$ estrias em $10 \mu \mathrm{m}$. Habitat: salobro.

Primeira citação de ocorrência da espécie para o Estado.

Material examinado: BRASIL. Rio Grande do Sul: Rio Grande, laguna dos Patos, (HAS 107874, lâm n. 6056); (HAS 107851, lâm n. 6067); (HAS 107853, lâm n. 6071).

\section{Amphora pseudoholsatica Nagumo \& Kobaysi, 1990}

Fig. 4C

Medidas: comprimento 16-38 $\mu \mathrm{m}$; largura 4-7,5 $\mu \mathrm{m} ; 10$ a11 estrias em $10 \mu \mathrm{m}$. Habitat: salobro.

Primeira citação de ocorrência da espécie para o Estado.

Material examinado: BRASIL. Rio Grande do Sul: Rio Grande, laguna dos Patos, (HAS 107873, lâm n. 6044); (HAS 107850, lâm n. 6061, 6062); (HAS 107851, lâm n. 6067); (HAS 107853, lâm n. 6070).

Ordem Bacillariales

Família Bacillariaceae

Bacillaria paxillifer (Müller) Hendey, 1951

Fig. 4D

Medidas: comprimento 101-110 $\mu \mathrm{m}$; largura 6-6,5 $\mu \mathrm{m}$ de largura. Habitat: salobro.

Material examinado: BRASIL. Rio Grande do Sul: Rio Grande, laguna dos Patos, (HAS 107850, lâm n. 6061); (HAS 107851, lâm n. 6067); (HAS 107853, lâm n. 6071).

\section{Hantzschia amphioxys (Ehrenberg) Grunow, 1880}

Fig. 4E

Medidas: comprimento 27-40 $\mu \mathrm{m}$; largura 4-7 $\mu \mathrm{m}$ de largura. Habitat: salobro.

Material examinado: BRASIL. Rio Grande do Sul: Rio Grande, laguna dos Patos, (HAS 107874, lâm n. 6056, 6058).

\section{Tryblionella apiculata Gregory, 1857}

Fig. 4F

Medidas: comprimento 47-62 $\mu \mathrm{m}$; largura 7,5-9 $\mu \mathrm{m}$; 14-15 estrias em $10 \mu \mathrm{m}$. Habitat: salobro.

Material examinado: BRASIL. Rio Grande do Sul: Rio Grande, laguna dos Patos, (HAS 107873, lâm n. 6044, 6048); (HAS 107850, lâm n. 6063); (HAS 107851, lâm n. 6067).
Tryblionella circunsuta (Bailey) Ralfs, 1861

Fig. 4G

Medidas: comprimento 143-1 Van Heurck $66 \mu \mathrm{m}$; largura 58-60 $\mu \mathrm{m}$ de largura. Habitat: salobro.

Material examinado: BRASIL. Rio Grande do Sul: Rio Grande, laguna dos Patos, (HAS 107873, lâm n. 6045, 6048).

Tryblionella perversa Grunow, 1990

Fig. $4 \mathrm{H}$

Medidas: comprimento 33-38 $\mu \mathrm{m}$; largura $16 \mu \mathrm{m}$ de largura. Habitat: marinho e salobro.

Material examinado: BRASIL. Rio Grande do Sul: Rio Grande, laguna dos Patos, (HAS 107853, lâm n. 6070, 6071).

\section{Tryblionella victoriae Grunow, 1862}

Fig. 4I

Medidas: comprimento 36-37 $\mu \mathrm{m}$; largura $16 \mu \mathrm{m} ; 6$ fíbulas em $10 \mu \mathrm{m} ; 6$ estrias em $10 \mu \mathrm{m}$. Habitat: marinho.

Material examinado: BRASIL. Rio Grande do Sul: Rio Grande, laguna dos Patos, (HAS 107873, lâm n. 6045); (HAS 107850, lâm n. 6063); (HAS 107853, lâm n. 6070).

Nitzschia filiformis (Smith) Van Heurck, 1896

Fig. 4J

Medidas: comprimento 46-80 $\mu \mathrm{m}$; largura 4-6 $\mu \mathrm{m}$; 7-8 fíbulas em $10 \mu \mathrm{m} ; 30-33$ estrias em $10 \mu \mathrm{m}$. Habitat: salobro.

Material examinado: BRASIL. Rio Grande do Sul: Rio Grande, laguna dos Patos,

Nitzschia prolongata Hustedt, 1938

Fig. $4 \mathrm{~K}$

Medidas: comprimento 79-120 $\mu \mathrm{m}$; largura 4-6 $\mu \mathrm{m}$; 6-7 fíbulas em $10 \mu \mathrm{m} ; 20-22$ estrias em $10 \mu \mathrm{m}$. Habitat: salobro.

Primeira citação de ocorrência da espécie para o Estado.

Material examinado: BRASIL. Rio Grande do Sul: Rio Grande, laguna dos Patos, (HAS 107873, lâm n. 6045, 6048); (HAS 107850, lâm n. 6061).

Nitzschia rhopalodioides Hustedt, 1955

Fig. 4L

Medidas: comprimento $64 \mu \mathrm{m}$; largura $10 \mu \mathrm{m}$; 5 fíbulas em $10 \mu \mathrm{m}$. Habitat: marinho.

Primeira citação de ocorrência da espécie para o Estado.

Material examinado: BRASIL. Rio Grande do Sul: Rio Grande, laguna dos Patos, (HAS 107850, lâm n. 6062).

Nitzschia scalpelliformis (Grunow) Grunow, 1880

Fig. 4M

Medidas: comprimento 39-98 $\mu \mathrm{m}$; largura 4,5-5 $\mu \mathrm{m}$; 8-9 fíbulas em $10 \mu \mathrm{m} ; 22-25$ estrias em $10 \mu \mathrm{m}$. Habitat: salobro.

Material examinado: BRASIL. Rio Grande do Sul: Rio Grande, laguna dos Patos, (HAS 107850, lâm n. 6061, 6062); (HAS 107853, lâm n. 6070). 
Nitzschia subcohaerens var. scotica (Grunow) Van Heurck, 1896

Fig. 4N

Medidas: comprimento $40 \mu \mathrm{m}$; largura $5 \mu \mathrm{m} ; 8$ fíbulas em $10 \mu \mathrm{m} ; 30$ estrias em $10 \mu \mathrm{m}$. Habitat: salobro.

Material examinado: BRASIL. Rio Grande do Sul: Rio Grande, laguna dos Patos, (HAS 107873, lâm n. 6045).

\section{Denticula kuetzingii Grunow, 1862}

Fig. $4 \mathrm{O}$

Medidas: comprimento 11-12 $\mu \mathrm{m}$; largura $3 \mu \mathrm{m} ; 87$ estrias em $10 \mu \mathrm{m}$. Habitat: dulcícola.

Primeira citação de ocorrência da espécie para o Estado.

Material examinado: BRASIL. Rio Grande do Sul: Rio Grande, laguna dos Patos, (HAS 107874, lâm n. 6056); (HAS 107853, lâm n. 6071).

Ordem Rhopalodiales

Família Rhopalodiaceae

Epithemia adnata (Kützing) Brébisson, 1838

Fig. 4P

Medidas: comprimento 46-55 $\mu \mathrm{m}$; largura 8-9 $\mu \mathrm{m}$; 6 costas em $10 \mu \mathrm{m} ; 6$ estrias em $10 \mu \mathrm{m}$. Habitat: salobro e dulcícola.

Material examinado: BRASIL. Rio Grande do Sul: Rio Grande, laguna dos Patos, (HAS 107851, lâm n. 6066, 6067); (HAS 107853, lâm n. 6070).

\section{Rhopalodia brebissonii Krammer, 1987}

Fig. 4Q

Medidas: comprimento 23-33 $\mu \mathrm{m}$; largura 5-7 $\mu \mathrm{m}$; 3 costas em $10 \mu \mathrm{m}$. Habitat: salobro.

Material examinado: BRASIL. Rio Grande do Sul: Rio Grande, laguna dos Patos, (HAS 107850, lâm n. 6061, 6062); (HAS 107851, lâm n. 6067).

\section{Rhopalodia rumrichae Krammer, 1987}

Fig. 4R

Medidas: comprimento 21-33 $\mu \mathrm{m}$; largura 9-13 $\mu \mathrm{m} ; 3$ costas em $10 \mu \mathrm{m}$. Habitat: dulcícola.

Primeira citação de ocorrência da espécie para o Estado.

Material examinado: BRASIL. Rio Grande do Sul: Rio Grande, laguna dos Patos, (HAS 107873, lâm n. 6044, 6048); (HAS 107850, lâm n. 6062); (HAS 107851, lâm n. 6067); (HAS 107853, lâm n. 6070).

Ordem Surirellales

Família Surirellaceae

Surirella rorata Frenguelli, 1935

Fig. 4S

Medidas: comprimento 80-168 $\mu \mathrm{m}$; largura 45-96 $\mu \mathrm{m}$; 11 projeções aliformes em $100 \mu \mathrm{m}$. Habitat: marinho e salobro.
Material examinado: BRASIL. Rio Grande do Sul: Rio Grande, laguna dos Patos, (HAS 107873, lâm n. 6046, 6049); (HAS 107851, lâm n. 6065).

A comunidade esteve composta por $60 \%$ de diatomáceas salobras, $24 \%$ marinhas e $16 \%$ dulcícolas. A representatividade das espécies de habitat salobro demonstrou uma comunidade adaptada à variação de salinidade das marismas.

Nos trabalhos realizados em outros ambientes estuarinos brasileiros e sul-americanos foram citados 20 táxons (40\%) registrados nas marismas do estuário da laguna dos Patos, quais sejam: Cyclotella meneghiniana, Nitzschia scalpelliformis (como Nitzschia obtusa var. scalpelliformis), Paralia sulcata e Terpsinoë americana para o estuário do Rio Paripe - Pernambuco (Moura et al., 2007); Catenula adhaerens, Nitzschia scalpelliformis e Tabularia fasciculata (Sylvestre et al., 2001) para a Lagoa de Araruama - Rio de Janeiro; Diploneis smithii, Hantzschia amphioxys e Paralia sulcata (como Melosira sulcata) para a Baía Sul Santa Catarina (Mosimann, 1988); Achnanthes curvirostrum, Capartogramma crucicula, Cyclotella striata, Dimeregramma minor, Diploneis didyma, Diploneis smithii, Hantzschia amphioxys, Mastogloia pumila, Paralia sulcata, Terpsinoë americana, Tryblionella circunsuta (como Nitzshia circunsuta) e Tryblionella perversa (como Nitzschia perversa) para o estuário do Rio Ratones - Santa Catarina (Fernandes et. al., 1990); Actinoptychus senarius, Cyclotella meneghiniana, Cyclotella striata, Navicula peregrina, Paralia sulcata e Terpsinoë americana para o Complexo estuarino Tramandaí-Armazém (Rosa \& Callegaro, 1988; Rosa \& Werner, 1993; Rosa et al., 1994); Actinoptychus senarius, Cocconeis placentula, Cyclotella meneghiniana, Cyclotella striata, Hantzschia amphioxys, Navicula peregrina, Nitzschia filliformis e Rhopalodia brebissoni para o estuário do Río de la Plata Argentina e Uruguai (Licursi et al., 2006).

Para os ambientes de marismas da Inglaterra (Round, 1960), Estados Unidos da América (Sullivan 1975, 1982; Sherrod, 1999), Dinamarca (Szkornik et al.,2006) e Canadá (Roe et al., 2009), que podem ser considerados ambientes homólogos as marismas do sul do Brasil foram citados também 20 táxons (40\%) aqui registrados, quais sejam: Actinoptychus senarius, Bacillaria paxillifer (como Bacillaria paradoxa), Cocconeis placentula, Cyclotella meneghiniana, Cyclotella striata, Cymatosira belgica, Dimeregramma minor, Diploneis didyma, Diploneis smithii, Fallacia oculiformis (como Navicula oculiformis), Frustulia creuzburgensis, Hantzschia amphioxys, Mastogloia pumila, Navicula peregrina, Nitzschia filliformis, Nitzschia scalpelliformis (como Nitszchia obtusa var. scalpelliformis), Paralia sulcata (como Melosira sulcata), Staurophora salina (como Stauroneis salina), Tabularia fasciculata (como Fragilaria fasciculata, Synedra tabulata e Synedra fasciculata), Tryblionella perversa (como Nitzschia perversa).

Em relação ao número de espécies, os gêneros mais bem representados foram Nitzschia (5 spp.) e Tryblionella (4 spp.). 


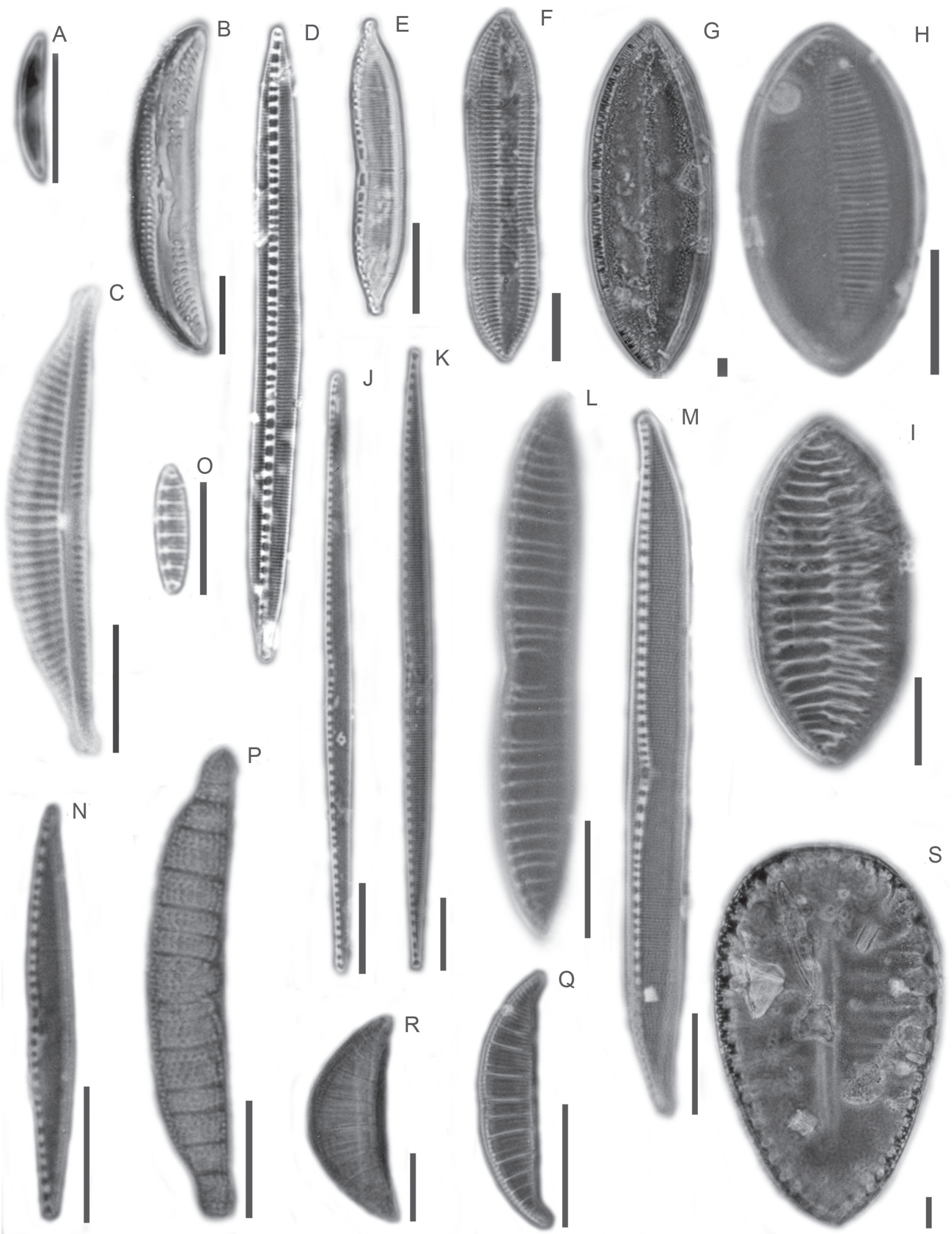

Figura 4. A. Catenula adhaerens (Mereschkowsky) Mereschkowsky. B. Amphora crucifera Cleve. C. Amphora pseudoholsatica Nagumo \& Kobaysi. D. Bacillaria paxillifer (Müller) Hendey. E. Hantzschia amphioxys (Ehrenberg) Grunow. F. Tryblionella apiculata Gregory. G. Tryblionella circunsuta (Bailey) Ralfs. H. Tryblionella perversa Grunow. I. Tryblionella victoriae Grunow. J. Nitzschia filiformis (Smith) Van Heurck. K. Nitzschia prolongata Hustedt. L. Nitzschia rhopalodioides Hustedt. M. Nitzschia scalpelliformis (Grunow) Grunow. N. Nitzschia subcohaerens var. scotica (Grunow) Van Heurck. O. Denticula kuetzingii Grunow. P. Epithemia adnata (Kützing) Brébisson. Q. Rhopalodia brebissonii Krammer. R. Rhopalodia rumrichae Krammer. S. Surirella rorata Frenguelli. Barra $=10 \mu \mathrm{m}$. 
A classe mais bem representada foi Bacillariophyceae com 40 táxons; seguida por Coscinodiscophyceae com 8 táxons e Fragilariophyceae com 2 táxons. As ordens mais bem representadas foram Naviculales com 13 táxons e Bacillariales com 12 táxons. As famílias com maior número de espécies foram Bacillariaceae com 12 spp. e Naviculaceae com 6 spp.

A riqueza estimada para a comunidade foi de 53,5 espécies, com intervalo de confiança entre 45 e 62 espécies. A riqueza observada neste levantamento, 50 espécies, está dentro do intervalo de valores esperados indicando que o esforço amostral foi adequado. A distribuição dos táxons encontra-se na Tabela 2.
Diferenças na composição de diatomáceas dos cinco pontos amostrados eram esperadas, tanto por cada um representar uma comunidade espacialmente distante do outro, quanto por dois destes pontos haverem sido amostrados temporalmente distantes dos outros três. Estas hipóteses foram avaliadas através da análise da diversidade- $\beta$.

A diversidade- $\beta$ para este levantamento tem valor mínimo 1,0 e máximo 5,5 e o valor calculado 1,05 indicou pequena variação. Este resultado demonstra que a comunidade estava bem distribuída e semelhante na área de estudo.

Tabela 2. Distribuição das diatomáceas nos pontos amostrados nas marismas do estuário da laguna dos Patos (+ indicando presença, - indicando ausência) em janeiro de 2002 e março de 2008.

\begin{tabular}{|c|c|c|c|c|c|}
\hline Táxon & Estação 1 & Estação 2 & Estação 3 & Estação 4 & Estação 5 \\
\hline Achnanthes curvirostrum & - & - & + & + & + \\
\hline Achnanthes parvula & + & + & - & - & - \\
\hline Actinoptychus senarius & + & - & - & - & - \\
\hline Amphora crucifera & - & + & - & + & + \\
\hline Amphora pseudoholsatica & + & - & + & + & + \\
\hline Bacillaria paxillifer & - & - & + & + & + \\
\hline Caloneis bivittata var. lata & + & - & - & - & - \\
\hline Capartogramma crucicula & - & - & - & - & + \\
\hline Catenula adhaerens & + & + & + & - & + \\
\hline Chamaepinnularia truncata & - & - & + & - & - \\
\hline Cocconeis disculoides & + & - & + & - & + \\
\hline Cocconeis placentula var. lineata & - & - & + & + & + \\
\hline Cyclotella meneghiniana & + & - & - & + & + \\
\hline Cyclotella striata & - & - & - & + & + \\
\hline Cymatosira belgica & - & + & - & - & - \\
\hline Cymbella grossestriata & + & - & + & + & - \\
\hline Denticula kuetzingii & - & + & - & - & + \\
\hline Diploneis didyma & + & - & - & + & + \\
\hline Diploneis smithii & + & + & + & + & + \\
\hline Dimeregramma minor & - & + & + & - & - \\
\hline Epithemia adnata & - & - & - & + & + \\
\hline Fallacia oculiformis & - & - & + & - & - \\
\hline Frustulia creuzburguensis & - & - & + & - & - \\
\hline Hantzschia amphioxys & - & + & - & - & - \\
\hline Hippodonta hungarica & - & - & - & + & + \\
\hline Luticola goeppertiana & + & + & + & + & + \\
\hline Martyana martyi & + & + & - & - & + \\
\hline Mastogloia pumila & - & - & + & + & - \\
\hline Navicula peregrina & - & - & + & + & + \\
\hline Navicula recens & + & + & + & + & + \\
\hline Nitzschia filiformis & + & - & + & - & - \\
\hline Nitzschia prolongata & + & - & + & - & - \\
\hline Nitzschia rhopalodioides & - & - & + & + & - \\
\hline Nitzschia scalpelliformis & - & - & + & - & + \\
\hline Nitzschia subcohaerens var. scotica & + & - & - & - & - \\
\hline Paralia sulcata & + & + & - & - & - \\
\hline
\end{tabular}


Tabela 2. Continuação.

\begin{tabular}{|c|c|c|c|c|c|}
\hline Táxon & Estação 1 & Estação 2 & Estação 3 & Estação 4 & Estação 5 \\
\hline Parlibellus crucicula & + & - & + & - & - \\
\hline Placoneis abundans & - & - & - & - & + \\
\hline Pleurosira laevis & + & - & - & + & - \\
\hline Rhopalodia brebissoni & - & - & + & + & - \\
\hline Rhopalodia rumrichiae & + & + & + & + & + \\
\hline Seminavis strigosa & - & - & + & + & + \\
\hline Staurophora salina & - & - & + & + & + \\
\hline Surirella rorata & + & - & - & + & + \\
\hline Tabularia fasciculata & + & - & - & + & - \\
\hline Terpsinoë americana & + & - & + & - & + \\
\hline Tryblionella apiculata & + & - & + & + & + \\
\hline Tryblionella circunsuta & + & - & - & + & - \\
\hline Tryblionella perversa & - & - & - & - & + \\
\hline Tryblionella victoriae & + & - & + & - & + \\
\hline
\end{tabular}

\section{Agradecimentos}

À Dra Svetlana Medeanic, do Centro de Estudos Costeiros, Instituto de Geociências da Universidade Federal do Rio Grande do Sul pelo fornecimento de amostras e apoio nas coletas. Ao professor Jorge Luiz Waechter do Departamento de Botânica da Universidade Federal do Rio Grande do Sul pelos esclarecimentos em ecologia vegetal. À Fundação de Amparo a Pesquisa no Rio Grande do Sul - FAPERGS e ao Conselho Nacional de Desenvolvimento Científico e Tecnológico (CNPq) pela concessão de bolsa aos autores (Proc. 08511147 e 07511644 ).

\section{Referências bibliográficas}

Battarbee, R.W. 1986. Diatom analysis.Pp. 527-570. In: Berglund B.E. (ed.), Handbook of Holocene Palaeoecology and Palaeohydrology. Toronto, John Wiley and Sons.

Buselato-Toniolli, T. 1986. Diatomoflórula (Bacillariophyceae) associada à Hypena musciformis (Wulfen) Lamouroux (Rhodophyceae) do Litoral de Torres, Rio Grande do Sul, Brasil. Iheringia, Série Botânica 35: 65-126.

Chao, A. 1987. Estimating the population size for capture-recapture data with unequal catchability. Biometrics 43: 783-791.

Colwell, R.K. 2006. EstimateS: Statistical estimation of species richness and shared species from samples. Version 8.0. User's Guide and application published at: htttp://purl.oclc.org/estimates.

Cordazzo, C.V. \& Seeliger, U. 1995. Guia ilustrado da vegetação costeira do extremo sul do Brasil. Rio Grande, FURG.

Danielidis, D.B. \& Mann, D.G. 2003. New species and new combinations in the genus Seminavis (Bacillariopyta). Diatom Research 18(1): 21-39.

Fernandes, L.F.; Souza-Mosimann, R.M. \& Fernandes, G.F. 1990. Diatomáceas (Bacillariophyceae) do Rio Ratones, Florianópolis, Santa Catarina, Brasil I - Baixo Curso e Estuário. Ínsula 20: 111-112.

Frenguelli, J. 1929. Diatomee fossilidelle conche saline del deserto chilenoboliviano. Bolletino della Società Geologica Italiana 47: 185-236.

Frenguelli, J. 1935. Diatomeas de la Mar Chiquita al Norte del Mar del Plata (Provincia de Buenos Aires). Notas del Museo de La Plata, Botánica 5:121-140.

Frenguelli, J. 1938. Diatomeas del Querandinense estuariano del rio Matanza en Buenos Aires, Revista del Museo de La Plata 1(5): 291-326.

Hartley, B. 1996. An Atlas of British Diatoms. Bristol, Biopress.

Hendey, N.I. 1964. An introductory account of the smaller algae of British coastal waters. Part V. Bacillariophyceae (Diatoms). Pp. 317. In: Fishery Investigations Series, vol. 4, London, Koeltz Scientific Books Koenigstein.
Hustedt, F. 1930. Bacillariophyta (Diatomeae), (Die Susswasser-Flora Mitteleuropas). G. Fisher Ed. A. Pasher.

Hustedt, F. 1937. Systematische und ökologische untersuchungen über die diatomeen-flora von Java, Bali und Sumatra. Archiv für Hydrobilogie 15(2):187-295

Hustedt, F. 1927-66. Die Kieselalgen Deutschlands, Osterrreichs und der Schweiz. In Dr. L Rabenhorst's Kryptogamen-Flora von Deutschland, Osterreich und der Schweiz, Akademische Verlagsgesellschaft Leipzig. 3 vols.

Krammer, K. \& Lange-Bertalot, H. 1985. Naviculaceae. Berlin, J.Cramer. (Bibliotheca Diatomologica, 9).

Krammer, K. \& Lange-Bertalot, H. 1986. Susswasserflora von Mitteleuropa. Bacillariophyceae 1. Teil: Naviculaceae. Stuttgart, Gustav Fischer.

Krammer, K. \& Lange-Bertalot, H. 1988. Susswasserflora von Mitteleuropa. Bacillariophyceae 2. Teil: Bacillariaceae, Epithemiaceae, Surirellaceae. Stuttgart, Gustav Fischer.

Lange-Bertalot, H. 1998. Tropical Diatoms of South America I. A.R.G. Gantner. (Iconographia Diatomologica, 5).

Lange-Bertalot, H. \& Metzeltin, D. 2001. Diatoms of Europe. Navicula sensu stricto. 10 Genera Separated from Navicula sensu lato. Frustulia. A.R.G. Gantner. (Diatoms of the European Inland Waters and Comparable Habitats, 2).

Licursi, M; Sierra, M.V. \& Gómez, N. 2006. Diatom assemblages from a turbid coastal plain estuary: Río de la Plata (South America). Journal of Marine Systems 62: 35-45

Metzeltin, D. \& Witkowski, A. 1996. Diatomeen der Bären-Insel. A.R.G. Gantner. (Iconographia Diatomologica, 4).

Metzeltin, D.; Lange-Bertalot, H. \& García-Rodriguez, F. 2005. Diatoms from Uruguay. A.R.G. Gantner. (Iconographia Diatomologica, 15).

Moreira-Filho, H.; Valente-Moreira, I.M.; Souza-Mosimann, R.M. \& Cunha, J.A. 1990. Avaliação florística e ecológica das diatomáceas (ChrysophytaBacillariophyceae) marinhas e estuarinas nos estados do Paraná, Santa Catarina e Rio Grande do Sul. Estudos Biológicos 25: 5-48.

Mosimann, R.M., 1988. Estudo preliminary das diatomáceas (ChrysophytaBacillariophyceae) da Baía Sul, Florianópolis, Santa Catarina - Brasil. Ínsula 18: 23-74.

Moura, A.; Bittencourt-Oliveira, M. \& Nascimento, E. 2007. Benthic Bacillariophyta of Paripe river estuary in Pernambuco state, Brazil. Brazilian Journal of Biology 67(3): 393-401.

Niencheski, L.F. \& Windom, H.L. 1994. Nutrient flux and budget in Patos Lagoon Estuary. The Science Total Environment 149: 53-60.

Patrick, R. \& Reimer, C.W. 1966. The diatoms of the United States, exclusive of Alaska and Hawaii. Philadelphia, Livingstgon Company.

Roe, H.M.; Doherty, C.T.; Patterson, R.T. \& Swindles, G.T. 2009. 
Contemporary distributions of saltmarsh diatoms in the SeymourBelize Inlet Complex, British Columbia, Canada: Implications for studies of sea-level change. Marine Micropaleontology 70: 134-150.

Rosa, Z.M. 1982. Diatomáceas marinhas e estuarinas de Tramandaí, Rio Grande do Sul, Brasil. Iheringia. Série Botânica 29: 49-145.

Rosa, Z.M. \& Callegaro, V.L.M. 1988. Diatomáceas da Lagoa de Tramandaí e da Lagoa do Armazém, Rio Grande do Sul, Brasil: I - Gênero Navicula Bory. Iheringia. Série Botânica 37: 17-32.

Rosa, Z.M. \& Werner, V.R. 1993. Diatomáceas da Lagoa de Tramandaí e da Lagoa do Armazém, Rio Grande do Sul, Brasil: II - Gêneros Gyrosigma Hassal, Pleurosigma W.Smith e Mastogloia Thwaites. Iheringia. Série Botânica 43: 67-87.

Rosa, Z.M.; Werner, V.R. \& Dacroce, L. 1994. Diatomáceas da Lagoa de Tramandaí e da Lagoa do Armazém, Rio Grande do Sul, Brasil: III Ordem Centrales. Iheringia. Série Botânica 48: 29-55.

Round, F.E. 1960. The diatom flora of a salt marsh on the River Dee. New Phytologist 59: 332-348.

Round, F.E.; Crawford, R.M. \& Mann, D.G. 1990. The diatoms: Biology \& Morphology of the Genera. New York, Cambridge University Press.

Seeliger, U. \& Costa, C.S.B. 1998. Impactos naturais e humanos. In: Os Ecossistemas Costeiro e Marinho do Extremo Sul do Brasil (Seeliger, U., Odebrecht, C. \& Castello, J.P. Eds.). Rio Grande, Editora Ecoscientia.

Sherrod, B.L. 1999. Gradient analysis of diatom assemblages in a Puget Sound salt marsh: can such assemblages be used for quantitative paleoecological reconstructions? Palaeogeography, Palaeoclimatology, Palaeoecology 149: 213-226.

Siver, P.A.; Hamilton, P.B.; Stachura-Suchoples, K. \& Kociolek, J.P. Diatoms of North America. The Freshwater Flora of Cape Cod, Massachusetts, USA. A.R.G. Gantner. (Iconographia Diatomologica, 14).

Snoeijs, P.J.M. 1992. Studies in the Tabularia fasciculata complex. Diatom
Research 7: 313-344.

Snoeijs, P.J.M.; Hällfors, G. \& Leskinen, E. 1991. The transfer of two epipsamic diatom species to the genus Martyana. Diatom Research 6: $165-173$.

Sullivan, M.J. 1975. Diatom Communities from a Delaware Salt Marsh. Journal of Phycology 11: 384-390.

Sullivan, M.J. 1982. Distribution of edaphic diatoms in a Mississippi Salt Marsh: A canonical correlation Analysis. Journal of Phycology 18: 130-133.

Sündback, K. \& Medlin, L.K. 1986. A light and electron microscopic study of the epipsammic diatom Catenula adhaerens Mereschkowsky. Diatom Research 1: 283-290.

Sylvestre, F.; Beck-Eichler, B.; Duleba, W. \& Debenay, J. 2001. Modern Bethic diatom distribution in a hypersaline coastal lagoon: the Lagoa de Araruama (R.J.), Brazil. Hydrobiologia 443: 213-231.

Szkornik, K.; Gehrels, W.R. \& Kirby, J.R. 2006. Salt-marsh diatom distributions in Ho Bugt (western Denmark) and the development of a transfer function for reconstructing Holocene sea-level changes. Marine Geology 235: 137-150.

Van Der Werff, A. 1955. A new method of concentrating and cleaning diatoms and others organisms. Verhaudlungen der Internationalen Vereinigung der Limnologie 55: 276-277.

Van Heurck, H. 1896. Synopsis des Diatomées de Belgique. Anvers, Edite par l'Auteur.

Villwock, J.A.; Tommazelli, L.J.; Loss, E.L.; Horn N.H.; Bachi, F.A. \& Dehnart, B.A. 1986. Geology of the Rio Grande do Sul coastal province. Pp. 79-97. In: Rabassa J. (ed.) Quaternary of South America Antarctica Peninsula, v. 4. Balkema, Rotterdam.

Witkowski, A.; Lange-Bertalot, H. \& Metzeltin, D. 2000. Diatom Flora of Marine Coasts: I. A.R.G. Gantner. (Iconographia Diatomologica, 7).

Whittaker, R.H. 1960. Vegetation of the Siskiyou Mountains, Oregon and California. Ecological Monographs 30: 279-338.

Whittaker, R.H. 1972. Evolution and measurement of species diversity. Taxon 21: 231-251. 